\title{
Guidance for chronic subdural hematoma surgery in the novel coronavirus pandemic (Covid-19)
}

\author{
Marcelo Moraes Valença, \\ Martina Falcão Valença ${ }^{3}$ (iD \\ Carolina Martins ${ }^{2,4}$ \\ Luciana Patrízia Alves Andrade-Valença ${ }^{1,2}$ \\ Marcelo Andrade Valença ${ }^{2}$ \\ Ana Cristina Veiga Silva ${ }^{2,4}$ \\ Hildo Rocha Cirne de Azevedo Filho ${ }^{5}$
}

${ }^{1}$ Unimed Recife, Recife, Pernambuco, Brazil

${ }^{2}$ Neurology and Neurosurgery Unit, Hospital das Clínicas, Federal University of Pernambuco, Recife, Brazil

${ }^{3}$ International Neuroscience Institute, Hanover, Germany, Unit of Neurosurgery

${ }^{4}$ Hospital Pelópidas Silveira, Recife, PE, Brazil

${ }^{5}$ Department of Neurosurgery, Hospital da Restauração, Universidade de Pernambuco, Recife, Pernambuco, Brazil.

\section{$\sum$}

Marcelo Moraes Valença Neurosurgery Unit, Hospital das Clinicas, Federal University of Pernambuco, Cidade Universitária, 50670-901 Recife, Pernambuco, Brazil.

mmvalenca@yahoo.com.br

This article was edited by:

Fernando Cruz

\section{Keywords:}

Chronic subdural hematoma

Covid-19

Prevention

Guidance

Neurosurgery

Treatment

\begin{abstract}
Of great concern to the neurosurgical community is this novel coronavirus pandemic 2019, a result of dissemination to all continents of the severe acute respiratory syndrome coronavirus 2 (SARS-CoV-2). Without proper care, there is a very high chance of being infected by the lethal SARS-CoV-2 during a routine neurosurgical procedure. SARS-CoV-2 infected a significant part of the healthcare workers, including neurosurgeons, responsible for direct care for patients with Covid-19, and several of those infected have already died. Drastic modifications should be made in the process the operating room is prepared, and in the attitudes taken by medical personnel to minimize a possible contact with the SARS-CoV-2. The novel coronavirus is present not only in the respiratory tract, but the virus may also be encountered in blood, stool, and CSF. In this article, the authors want to address the topic of how neurosurgeons can mitigate the chance of being infected with SARS-CoV-2 during a surgical procedure performed on a patient with Covid-19. For this, we chose a common condition in neurosurgery practice - and one of the simplest in its execution - the chronic subdural hematoma (CSDH). There is still no consensus on how to operate patients with CSDH. This article aimed to elaborate a protocol with effective and minimally invasive techniques to be performed in the patient with both CSDH and Covid-19, with the dual intention of protecting the surgeon from SARS-CoV-2 infection and optimizing the patient's chance of healing.
\end{abstract}




\section{Introduction}

This his novel coronavirus pandemic 2019, a result of dissemination to all continents of the severe acute respiratory syndrome coronavirus 2 (SARS-CoV-2), is of great concern to the neurosurgical community. The World Health Organization (WHO) introduced the term Covid-19 (the acronym of "coronavirus disease 2019) to designate the disorder caused by SARS-CoV-2. Without proper care there is a very high chance of being infected by the lethal SARS-CoV-21, 2 during a routine neurosurgical procedure. Particularly with transnasal access, using an endoscope and drills for lesions at the base of the skull, the chance of becoming infected is alarming. ${ }^{3}$

SARS-CoV-2 has infected a significant part of the healthcare workers responsible for direct care of patients with coronavirus infection, ${ }^{4}$ and several of those infected have already died. In this regard, we read in the non-academic media that several neurosurgeons were infected with SARS-CoV-2, leading to death. $^{5}$

Drastic modifications have to be made in the way in which the operating room is prepared ${ }^{6}$ and in the measures taken by medical personnel in an attempt to minimize a possible contact of the SARS-CoV-2 with the oral, nasal and ocular mucosa of the neurosurgeons and their team. ${ }^{7}$ The evidence suggests that SARS-CoV-2 is more easily transmissible and lethal than seasonal influenza. ${ }^{4}$ In addition, the virus can spread over a distance of up to 8 meters $^{8}$ to infect individuals in the vicinity of the patient with Covid-19 and can remain suspended in the air for hours. The novel coronavirus is present not only in the respiratory tract, including the nasopharyngeal and oropharyngeal areas, ${ }^{4}$ but also in the blood and stools. 9,10 Preliminary data suggests that the virus does not concentrate in the CSF. ${ }^{3}$ On the other hand, the virus has already been identified in the CSF in one patient."

In this article, the authors set out to address the topic of "how the neurosurgeons with the operating room staff can mitigate the chance of being infected with SARS-CoV-2 during a surgical procedure" performed on a patient with confirmed (or suspected) Covid-19. For this purpose, we chose a common condition in neurosurgery practice, and one of the simplest in its execution - chronic subdural hematoma (CSDH). ${ }^{12: 15}$

Patients with CSDH present clinically in different degrees of severity. ${ }^{12}$ This spectrum may vary from asymptomatic cases to those arriving in a comatose state in the emergency unit.

Despite being a simple technique compared to the extreme complexity of the majority of neurosurgical procedures, patients undergoing CSDH evacuation still have significant mortality. ${ }^{13,} 16-18$
It is incredible that even today, after more than a hundred years since neurosurgery was formally instituted, there is still no consensus on how we should operate patients with CSDH. ${ }^{19}$, ${ }^{20}$ There is still much doubt as to whether we should make one or two burr holes to drain the hematoma, use an intracranial drain to wash the cavity, leave a closed-system drainage in the postoperative period, ${ }^{13,21}$ use a subgaleal drain ${ }^{22}$, continue prolonged drainage ( $\geq 3$ days) in the postoperative period, ${ }^{23}$ perform a bedside percutaneous twist drill craniostomy, ${ }^{24}$ mini-craniotomy assisted by an endoscope, or whether we should perform a CT examination in the immediate postoperative period ${ }^{25}$ and when reoperating the patient on the basis only of neuroimaging, among other uncertainties. ${ }^{26}$

In 2005, a survey ${ }^{20}$ on neurosurgical practices in the treatment of CSDH was administered to Canadian neurosurgeons. The usual operatory techniques that the surgeons selected as the procedure of choice for the initial strategy to treat their patients were: one $(35.5 \%)$ or two $(49.5 \%)$ burr-hole craniostomy, craniotomy (4.7\%) or twist-drill craniostomy (9.3\%). Neither irrigation of the subdural cavity $(79.6 \%)$ nor the use of a subdural drain $\left(80.6 \%\right.$ ) were unanimous procedures..$^{20}$ In addition, antiepileptic drugs (82. $1 \%$ ) and corticosteroids (86.6\%) were used by the majority. ${ }^{20}$ There was no consensus among the surgeons on whether to keep patients supine following operation and maintain them routinely on antibiotics. The authors ${ }^{20}$ concluded that the "survey has identified variations in practice patterns among Canadian neurosurgeons".

Very recently, preliminary recommendations were suggested for use during a neurosurgery operation on Covid-19 infected patients.?

Thus, there are multiple treatment options ${ }^{20,27.33}$ and there is no consensus on the way a neurosurgeon decides how to best treat their patient with CSDH. The aim of this article was to elaborate a protocol with effective and minimally invasive techniques to be performed in the complex and fragile patient with both CSDH and Covid-19, with the dual intention of protecting the surgeon from SARS-CoV-2 infection and optimizing the patient's chance of being healed.

\section{Technique and guidance Surgical procedure}

The protocol elaborated here advocates drainage of the subdural hematoma through a single burr hole opening at the site of greatest thickness (maximum in-plane) of the hematoma. This burr hole is usually positioned in the center of the surface on which the hematoma is distributed in the supratentorial subdural space. The burr hole should be carefully made with the classic hand drill used in neurological surgery, and the assistant (i.e., scrub nurse) must irrigate continuously with saline to prevent aerosol formation. The use of a high-speed neuro- 
surgery drill should be avoided as it produces a dangerous droplet aerosol. ${ }^{3}$

The dura mater and the hematoma outer membranes are opened with the aid of a \#11 scalpel, and the dural edges are coagulated with the bipolar. Both dura mater and capsule must be opened in " + " format using the whole extension (diameter $\cong 0.8 \mathrm{~cm}$ ) of the bone gap (burr hole) so as to allow an effective leakage of the liquid hematoma.

After opening the subdural hematoma capsule (sometimes relatively thick) the surgeon must allow the liquid hematoma to escape, usually under great pressure, which occurs spontaneously for a few minutes. There is a liquid leakage, whose flow oscillates according to the cardiac cycle and concomitant variation of the intracranial pressure. When there is no more spontaneous ejection of liquid hematic material from the intracranial hematoma, the surgeon must use saline to gently wash the cavity, without inserting any drain through the cranial orifice. As there is a discrete aspiration force in diastole, the liquid usually enters the cavity and thus exits during systole, washing a little more residue of blood from the subdural hematoma. This washing continues until there is a return of clear liquid.

Meticulous bipolar hemostasis must be performed before applying surgical staples (the latter in order to save time) to close the 4-cm incision in the scalp. There is no need to close epicranial aponeurosis (galea). Every effort must be made to shorten the duration of the operation.

\section{Operating room and personal protective equipment}

Double surgical gloves are recommended for neurosurgeons to avoid infection due to glove ruptures and, at the end of the procedure, for safer removal of personal protective equipment (PPE).

Speaking by the surgical team must be restricted to what is essential to avoid contamination or movement of the mask on the face and possible entry of the viral particle.

Regarding protective equipment and Covid-19, the $C D C^{34}$ recommends the following:

Patients with suspected or confirmed Covid-19 may use the routine nonsterile, disposable isolation gowns used for patient care in healthcare settings. ${ }^{34}$

The healthcare personnel need to use surgical gowns (ANSI/ AAMI PB70 Levels 1-4) during surgical procedures. ${ }^{34}$ Gowns with minimal or low levels of barrier protection, e.g. ANSI/ AAMI PB70 Level 1 or 2, are not to be worn during surgical or invasive procedures or for medium-high risk contamination patient care activities. ${ }^{34}$

It is advisable to use coveralls designed to cover the whole body (360-degree protection, which includes the back and lower legs and sometimes also the head and feet), since surgical/isolation gowns do not effectively afford continuous whole-body protection. ${ }^{34}$

Hand hygiene before and after removing PPEs is imperative, using an alcohol-based hand sanitizer containing 60-95\% alcohol or, alternatively, washing hands with soap and water for at least $20 \mathrm{~s}$. When the hands are visibly soiled, soap and water should be used before using an alcohol-based hand sanitizer. ${ }^{34}$

Regarding the care of patients with Covid-19, very recent guidelines recommend ${ }^{4}$ the following:

Healthcare workers performing aerosol-generating procedures should use fitted respirator masks [N95 respirators (United States Federal Drug Agency standards), FFP2 (European standards-European Committee for Standards standards), or equivalent] with gloves, gown, and eye protection (face shield or safety goggles) (best practice statement). Surgical/medical masks are not recommended. Surgical/medical masks are intended to prevent contamination by large particles, droplets and sprays, but are less effective in blocking small particle aerosols $(<5 \mu \mathrm{m}) .{ }^{4}$ Here it is worth emphasizing that, in Wuhan, N95 masks used by ENT surgeons did not prevent infection during endonasal procedures. ${ }^{3}$

The Society of British Neurological Surgeons ${ }^{3}$ recommends preoperative Covid-19 testing in selected pituitary patients when surgery cannot be postponed and suggests other access routes rather than the transsphenoidal endonasal route: (1) Craniotomy or (2) Microscope-based trans-sphenoidal surgery, with a submucosal approach and entry to the sella using non-drill techniques. $^{3}$ The surgical procedures performed through the calvaria have a lower risk of contamination, except for frontal sinus opening or mastoid air cells violation.

Powered air-purifying respirators (PAPRs) can be used by healthcare workers when N95 is not available. ${ }^{4}$

The N95 respirator with an exhalation valve provides the same protection as N95 without a valve. ${ }^{34}$ The advantage of an exhalation valve is that it reduces exhalation resistance. ${ }^{34}$ Nevertheless, respirators with exhalation valves should not be utilized in circumstances in which a sterile field is imperative, such as a surgical procedure. ${ }^{34}$ The exhalation valve permits unfiltered exhaled air to contaminate the sterile operative field. 
Aerosol-generating procedures should be performed in a negative pressure room (best practice statement). ${ }^{4}$ The aerosol-generating procedures are: endotracheal intubation, bronchoscopy, open suctioning, administration of nebulized treatment, manual ventilation before intubation, physical proning of the patient, disconnecting the patient from the ventilator, non-invasive positive pressure ventilation, tracheostomy and cardiopulmonary resuscitation. ${ }^{4}$

If the patient needs endotracheal intubation, it is advisable that the most experienced staff member should perform the procedure to reduce the number of attempts and the risk of transmission. ${ }^{4}$ Large respiratory droplets predominantly spread SARS-CoV-2. ${ }^{4}$ Intubation is a small-particle (less $5 \mu \mathrm{m}$ ) aerosol-generating procedure that increases the risk of transmission to healthcare workers, given their close contact with the patient's airway and respiratory secretions. ${ }^{4}$

It is suggested that an operating room with negative pressure environment be positioned at an isolated corner of the operating complex, with separate access. ${ }^{6}$ The same operating room and the same anesthesia machine will only be used for Covid-19 cases for the duration of the pandemic. ${ }^{6}$

As a technical information detail, a negative pressure is generated by a ventilation system that provides extra air to access an isolated room by differential pressure and, concomitantly, the air be exhausted to be filtered through a high-efficiency particulate air (HEPA) filter directly before recirculation. ${ }^{4,6}$

For aerosol-generating procedures, the staff needs to use a negative pressure room with a minimum of 12 air changes per hour or at least $160 \mathrm{~L} /$ second/patient in an environment with natural ventilation (WHO guidance on COVID-19). ${ }^{4}$

One of the suggestions of the authors is that engineers develop a specific device with negative pressure suction, where the cooperative patient would remain inside and be separated from the room environment where the surgeon, scrub nurse and the anesthetist would be. This could possibly be in the shape of a tent or a large bag, with transparency at the face, allowing communication between the anesthetist and the patient. There are commercially available portable HEPA filters (mechanical air filters), and they are a valid alternative to be used in the room for removal of submicron infectious particles from the air. This type of filter can reduce virus transmission. ${ }^{4}$

Moreover, the presence of unnecessary personnel in the room should be avoided. ${ }^{4}$ In the operating room, the suggested staff (four at most) are as follows: an anesthesiologist, a neurosurgeon, a scrub nurse and an operating room nurse.

Due to the low likelihood of a younger individual presenting the most severe form of the disease, either the youngest surgeon on the team or one who has had the coronavirus infection with testing showing an elevated $\lg G$ should be scheduled to per- form the procedure; or a physician already vaccinated. Such a surgeon will probably be already immune to this viral disease. For guidance on details as to how the anesthetist should take when treating a patient with Covid-19 read the articles by $\mathrm{Ti}$ et al. ${ }^{6}$, Peng et al. ${ }^{35}$ and Wax and Christian ${ }^{36}$.

After surgery, the staff have to shower before returning to their regular duties. ${ }^{6}$ Moreover, in this situation it is not only the doctor whose health is at risk, but also and especially those who live in close contact with him, such as the family.

Ti and coworkers ${ }^{6}$ also recommend that a hydrogen peroxide vaporizer be employed to decontaminate the operating room.

\section{Discussion}

In some asymptomatic patients (Markwalder score 0-1) 37 $^{37}$ with a slight deviation of the midline structures and a small volume of subdural hematoma, we might maintain a conservative approach and presumably merely observe the progression ("wait-and-see" or "wait and scan" policy). ${ }^{28}$ Many of these cases progress without symptoms, and the imaging follow-up shows spontaneous resolution over 2-5 months. We have to follow the patient with special care if the chosen treatment is conservative, bearing in mind that in some series half of CSDH cases tend to worsen and require surgical drainage when $\mathrm{CSDH}$ is managed by conservative treatment. ${ }^{38}$

Also, tranexamic acid, oral steroid, angiotensin converting-enzyme inhibitors, mannitol, platelet-activating factor receptor antagonist and atorvastatin have all been tried as conservative treatment modalities for $\mathrm{CSDH}$ with promising preliminary results. ${ }^{37}$ The use of atorvastatin, as an adjunct drug to surgery, also appears to reduce the rate of recurrence in the postoperative period of subdural hematoma. ${ }^{38}$ Corticosteroid has been used in conservative treatment and also as an adjuvant to surgical procedure. ${ }^{30,39}$

The use of corticosteroids is still polemical in Covid-19 because of their adverse effects. ${ }^{4}$ Regarding viral pneumonia in the ICU, evidence has shown an increase in viral shedding with corticosteroid treatment, probably influencing viral replication. ${ }^{4}$ Recent guidelines were against the routine use of systemic corticosteroids for respiratory failure in Covid-19, but corticosteroids may be used in the sicker population of Covid-19 with acute respiratory distress syndrome (in a lower dosage and shorter treatment courses). ${ }^{4}$

Middle meningeal artery embolization has recently been recommended in symptomatic CSDH. ${ }^{29}$ Regarding Covid-19, we still have to evaluate which of the procedures involves the least risk for the neurosurgeon: endovascular or open surgery. Local anesthesia seems to be a safe method to use during CSDH evacuation, particularly when performing surgery on a collaborating patient. ${ }^{33,}$ 40-43 Local anesthesia without any 
sedation is the most recommended way to prevent decreased respiratory capacity and thereby avoid the use of non-invasive ventilation because of its high risk of aerosolization. ${ }^{4}$

When sedating or using anesthetic drugs, the anesthetist must assess the action of the selected medications on myocardial, ${ }^{44}$ hepatic and renal functions, considering that the Covid-19 may impair the normal function of these organs..$^{45}$

Furthermore, there is a doubt as to how patients with oligosymptomatic CSDH will progress if they develop respiratory symptoms that indicate the use of a mechanical ventilator in intensive care unit or, alternatively, if they develop other complications of coronavirus infection such as hepatic and cardiovascular dysfunctions or sepsis. Should we operate these patients preventively, correcting the probable intracranial hypertension? In this regard, since we still do not have the best answer, we should choose conservative management.

Another item to be considered is whether the person who becomes infected and acquires the disease in a hospital setting with critically ill patients with Covid-19 has the same clinical spectrum as the patients who were infected in the community, or they will have a more severe form? This considering the possibility of a strain with a greater aggressive and lethal power as well as a greater viral load when the individual is being exposed and acquired the Covid-19 at the time of infection. The use of appropriate clothing by medical and support staff is critical to reducing the risk of infection. ${ }^{46}$

The prognosis probably is not the same after operating patients already symptomatic with Covid-19. In this regard, the preexistence of comorbidities causing a disturbance of consciousness influences the severity and outcomes of patients who have undergone surgical treatment of $\mathrm{CSDH} .{ }^{47}$

In a series with 605 operated patients with $\mathrm{CSDH},{ }^{16}$ the lethality rate during hospitalization was significant (7.4\%), and a complete recovery was witnessed in only $62.9 \%$ of them. The comorbidities were hypertension (59.0\%), cardiopathies $(36.0 \%)$, previous stroke $(23.6 \%)$, clotting $(27.2 \%)$ and coagulation (17.0\%) alterations. ${ }^{16}$ Many of these comorbidities, if not all, undermine the prognosis not only of the final surgical result of patients with $\mathrm{CSDH}^{16}$ but also of those with Covid-19.48

In a preliminary study, Lei and colleagues ${ }^{49}$ reported that in a series of patients who were unintentionally programmed for elective surgeries in the incubation period of Civid-19, surprisingly, 44.1\% needed ICU and mortality was high (i.e., $20.5 \%)$. They raised the hypothesis that there may have been a higher inflammatory response and a change in immune function precipitated by the surgical procedure. ${ }^{49}$ This suggests that operate patients in the incubation period is associated with a poor prognosis. Every patient who will be operated on during the coronavirus pandemic should be considered as potentially infected with Covid-19 since there is a prolonged interval (approximately five days) of viral shedding before the onset of symptoms and some precautions must be well valued. Thus, elective surgeries must be postponed when possible.

\section{Toward a simpler neurosurgical approach}

Despite being a relatively simple procedure, draining a CSDH can have significant complications, such as acute intracranial rebleeding (high-density in the cerebral parenchyma or subdural space on CT), 50 remote contralateral intraparenchymal hemorrhage, ${ }^{51}$ subdural empyema, ${ }^{51}$ epileptic seizure, ${ }^{51}$ cerebellar hemorrhage, ${ }^{26}$ postoperative hyperfusion syndrome, ${ }^{52}$ tension postoperative pneumocephalus, ${ }^{53}$ epidural hematoma, ${ }^{54}$ postoperative thromboembolic complications, ${ }^{55}$ and internal hemorrhagic pachymeningios ${ }^{56}$.

Evidence shows that there are no significant differences in favorable results, whether or not using only a perforation in the skull to drain the hematoma when compared with two openings separated from each other by a few centimeters of distance.

Although not yet systematically assessed, the present authors believe that morbimortality is also substantially related to complications that occur during the surgical procedure, such as: (1) infection due to the permanence of the drain, (2) damage to the cerebral parenchyma during the act of irrigating the subdural cavity or in (3) positioning or (4) permanence from the drain in direct contact with the cortex (including motor area), (5) arterial bleeding, that occurs after drainage, which is often only perceived late on.

The placement of a subdural drain was associated with injuries in the parenchymal brain tissue in $2.8 \%$ of the cases (Figure 1). ${ }^{22} \mathrm{~A}$ few rare cases of small contralateral intraparenchymal hemorrhage after $\mathrm{CSDH}$ drainage were described and the authors hypothesized that they were due to a CSF overdrainage through the postoperative subdural drain. ${ }^{19,51,57}$

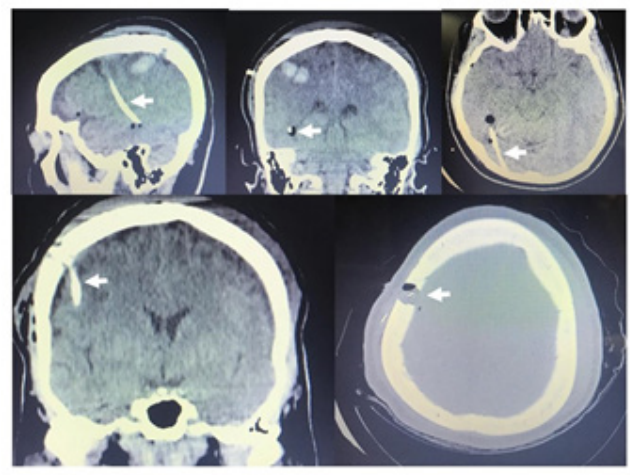

Figure 1. Postoperative computed tomography of a patient's skull operated on for a chronic subdural hematoma showing the catheter (white arrows) inside the brain parenchyma. Lower panels show the entrance of the catheter through a burr hole in the skull. 
In addition, it is not uncommon to observe surgeons washing the cavity with high hydraulic pressure when using the syringe attached to the drainage catheter. Of course, there may be hydrostatic shock on the parenchyma by the column of water injected intracranially.

Sixty patients with unilateral CSDH treated with burr-hole drainage underwent intraoperative irrigation with normal saline, and still $15 \%$ required additional surgery. ${ }^{58}$ Therefore, the use of irrigation with saline does not guarantee an effective procedure in all cases.

An interesting article was recently reported ${ }^{59}$ involving 1,252 patients operated for CSDH from three Scandinavian neurosurgical centers. The authors analyzed two groups: patients $<50$ years ( $n=52,4.2 \%$ ) and the remaining ones (i.e., $\geq 50$ years). Younger patients were more likely to present with headache $(86.5 \%$ vs. $37.9 \%)$ and vomiting ( $25 \%$ vs. $5.2 \%)$, while the $\geq 50$ years group more often presented with limb weakness ( $17.3 \%$ vs. $44.8 \%)$, speech impairment $(5.8 \%$ vs. $26.2 \%)$ and gait disturbance or falls (23.1\% vs. $50.7 \%$ ). The meta-analysis confirmed that younger patients are more likely to present more often signs of increased intracranial pressure such as headache, while the hemispheric symptoms are more likely in patients $\geq 50$ yr. There was no difference between the two groups in recurrence, overall complication rate and mortality within 90 days, suggesting that age had little influence.

Regarding the resolution of the subdural hematoma, a very interesting study ${ }^{60}$ showed the progression of 84 patients with unilateral CSDH. In non-recurrent cases of unilateral CSDH, the thickness of the residual hematoma cavity progressively decreased, and the residual CSDH cavity disappeared entirely by the 120 days postoperatively. ${ }^{60} \mathrm{~A}$ total disappearance of the hematoma cavity was verified in $45.6 \%$ of the individuals 3 months after surgery. ${ }^{60}$ In non-recurrent cases of unilateral $\mathrm{CSDH}$ there was a $49 \%$ reduction in hematoma (liquid) volume from $131.1 \pm 48.3 \mathrm{ml}$ to $66.5 \pm 33.3 \mathrm{ml} .{ }^{60}$ Considering the presence of air (volume of $19.8 \pm 19.3 \mathrm{ml}$ ), the subdural cavity one day after the surgical drainage of the hematoma was $86.3 \pm 39.9 \mathrm{ml}$; with a reduction of the subdural cavity by only $34 \%$, however with immediate improvement of the neurological picture in the vast majority of the operated patients. ${ }^{60}$ One day after the surgery there was a reduction in the following parameters: mean in-plane maximum thickness (from $25.6 \pm 5.8 \mathrm{~mm}$ to $14.9 \pm 5.1 \mathrm{~mm}$ ) and mean maximum midline displacement (from $9.4 \pm 4.3 \mathrm{~mm}$ to $5.7 \pm 2.3 \mathrm{~mm}$ ). ${ }^{60}$

Hematoma recurrence with reoperation shortly after drainage occurs in $7.6-33 \%$ of cases. ${ }^{13,50,60.63}$ depending on several potential causes, such as diabetes mellitus, ${ }^{19}$ elderly, high- and mixed-density of hematoma, leukemia, chronic renal failure, liver diseases, ${ }^{38}$ preexisting coagulopathy, and use of antiplatelet/anticoagulants agents. ${ }^{50,60,62,64 \cdot 67}$ Nonetheless, there are still doubts as to whether some of these factors would influence the recurrence rate.

Perhaps the rate of recurrence does not depend on the type of surgical procedure that was performed. A hypothesis that can be proposed is that the recurrence of the subdural hematoma that remains liquid was not because it was not drained, but it can be a particular characteristic of the disease. There is a re-accumulation of hematic fluid in the subdural space, in the same process as before the first surgery. In many cases, there is no report of head trauma. This idiopathic form of hematoma formation in the liquid state is not known how it started. The pathophysiology of CSDH remains poorly understood.

Really in large neurosurgery units with resident training, the resident is primarily responsible for the surgical treatment of patients with $\mathrm{CSDH}$, obviously with the supervision of a more experienced colleague. Thus, a more straightforward procedure to lower the chance of complications, by avoiding placing the drain in the subdural cavity, reducing possible sequelae or even death. ${ }^{68}$ We do believe that the surgery proposed in this article is valid for both patients with Covid-19 as well as for the regular patient without the infection.

Concerning the suggestion that indicate the younger surgeons to be chosen to perform the procedure, it is based on the surgical results from cases of patients with $\mathrm{CSDH}$ who were operated on by residents that were comparable to those obtained by the most experienced surgeon. ${ }^{69}$

There is no communication between the subdural space (physiologically a virtual space) and the subarachnoid compartment. Thus, there should be no CSF output after draining a $\mathrm{CSDH}$. If CSF is present in the postoperative drain, it was because there was a violation of the arachnoid membrane. Thus, CSF output should be interpreted as a complication of the surgical procedure. ${ }^{19}$ The outflow of large amounts of bloody CSF through the postoperative drain should cause disturbances in the intracranial CSF hydrodynamics and vascular dysregulation (both arterial and venous), with intracranial venous changes, causing vascular dilation of veins and sinuses.

Intracerebral hematoma after CSDH drainage was also reported, and the authors attributed the hemorrhage to vascular changes occurring after decompression and brain shifting. ${ }^{57}$ Can a drain with a particular negative pressure precipitate or contribute to the development of a postoperative hyperperfusion syndrome or intracerebral haemorrhage? We do not know yet.

The permanence of a drain that removes liquid from a transient "intracranial space" that will remain for several weeks regardless of the type of surgery performed can cause severe complications to our patients. For some reason not yet known, there is no immediate return of the cerebral hemisphere to its 
previous anatomical site. This process of reestablishing adaptive morphological changes in intracranial anatomical structures occurs over weeks. In the meantime, the space created by the hematoma remains, and through the internal pressure gradient difference, with less pressure in the subdural cavity, air, blood and other liquids are pulled. Due to high oncotic pressure, the absorption of water by the high amount of protein stored in the trapped liquid takes place.

Pavlov ${ }^{52}$ stressed that the complication rate of the surgical procedures to treat CSDH is more than $20 \%$, including recurrence of hematoma, worsening of the neurological status, postoperative delirium, among others. He suggests that a postoperative hyperperfusion syndrome (including postoperative delirium and neurological deterioration) develops after decompression of the hematoma in $51.4 \%$ of the cases. Similar to traumatic brain injury, a rapid decompression would cause a brain shifting.

Attempts to place drains in the hematoma cavity are a fearful maneuver, and many serious complications can happen in this surgical stage. Arterial bleeding can happen and, as a consequence, a transformation from a chronic hematoma into an acute one is a possibility. Penetration of the catheter into the cerebral parenchyma is another apprehensive inconvenience (Figure 1). These complications may occur mainly in younger patients when the subdural space may decrease rapidly with the drainage of the liquid hematoma.

The present authors believe that postoperative pneumocephalus $^{33}$ has no clinical repercussion, since always when opening the intracranial dura mater in a neurosurgical procedure, there will be the presence of air in the intracranial compartment, with progressive absorption occurring over 5-10 days. If that is a correct statement, there is no need to leave a drain in the cavity of the subdural space.

As the hematoma accumulates over several weeks, there is an adaptation of the cerebral parenchyma, allowing large volumes of the hematoma to expand, commonly without significant clinical repercussions until later on when focal neurological deficits or intracranial hypertension signs appear. There is probably a "retraction" (dehydration?) and displacement of the parenchyma and, even with the withdrawal of blood, the subdural space remains for several weeks allowing retention of liquid and ar. The presence of liquid inside the subdural space in the majority of the cases does seem not to compress vital structures. The presence of air means a negative pressure inside the cavity and, over time, the liquid will naturally replace air. The inexperienced surgeon who looks at a neuroimaging exam performed in the first days of the postoperative period may misinterpret it, judging it as a recurrence of the hematoma.

Many groups or surgeons prefer to use only one burr hole to operate patients with $\mathrm{CSDH} .{ }^{70}$ Some studies have shown that there is no significant difference between operating with one or two burr holes. A recent meta-analysis ${ }^{71}$ proves that there were no significant differences in recurrence rate, complication rate, and morbidity between single and double burr hole craniostomy in the treatment of $\mathrm{CSDH}$.

Single burr hole, double burr holes, or mini-craniotomy, when compared regarding the amount of drained hematoma $(71.7 \%$ vs. $73.7 \%$ vs. $71.9 \%$ ), and the postoperative air volume in the subdural space was practically the same. ${ }^{72}$

A study ${ }^{21}$ was done at Helsinki University Hospital with two groups of patients with CSDH: one with the position of a drain during the CSDH surgery and another without a drain. The recurrence rate was $18 \%$ in the non-drain group, and $6 \%$ in the drain group. The most interesting thing was that there was no effect on patient outcomes, infections, or other complications. ${ }^{21}$

The critical point is that we may have to reoperate a small group of patients, as in any other technique already used, 50, 73 but it is imperative to avoid preventable complications in any way. The authors believe that complications with the procedure are underreported. Even if, by performing the procedure suggested in this article, increases the reoperation rate, but without increasing complications and reduced morbidity and lethality rates are the ultimate goal. Studies must be carried out with methodological rigour adequately randomized to have the correct answer for the best way to operate a patient with CSDH. At the Hospital da Restauração (headed by one of the authors, HRCAF), Recife, the protocol used is to carry out two burr holes and placement of a drain for cases without Covid-19.

The authors do not consider it is necessary to use a catheter inside the hematoma cavity, or maintaining the drain in the postoperative period. The reduction of more than half (probably approximately $60-70 \%$ ) of the hematoma by spontaneous drainage would be enough to treat the patient with $\mathrm{CSDH}$, at least in most cases. The body will absorb the residue of the hematoma through an endogenous effective repair system operating over the next 3-6 months.

Perhaps the rate of recurrence does not depend on the type of surgical procedure that was performed. The recurrence of the liquefied subdural hematoma was not because it was not drained, but it can be a particular characteristic of the disease. The pathophysiology of CSDH remains poorly understood; the same can be said about the mechanism by which there is a local recurrence. There is a re-accumulation of hematic fluid in the subdural space, probably linked to a comparable process as before the first surgery.

In the case of the idiopathic CSDH, is it due to a spontaneous bleeding that starts a cascade of events until the formation of the capsule and expansion of the hematoma volume slowly and progressively. The hypotheses is that blood in the sub- 
dural space triggers inflammatory mechanisms that result in further small bleeding and water absorption. It is interesting to say that despite removing all the liquid from the CSDH by minimally invasive procedures, even so, the hematoma capsule remains. It may be that the hematoma capsule itself, closely linked to the dura mater, is the generator of the hematoma. Interesting is the fact that occlusion of the middle meningeal artery causes regression of the hematoma, suggesting an intimate relationship between the capsule and irrigation of the dura mater. Possibly, there would be a significant decrease or absence of recurrence of the hematoma if the capsule was removed entirely, but a craniotomy for that purpose increases the risk of further complications and would not benefit the vast majority of patients.

There is an idea that factors existing before surgery are related to a higher rate of reoperation in the example of patients with CSDH. ${ }^{50}$ This suggests that, at least in part, it is not the type of surgical approach that is linked with recurrence. It is often not a failure of surgical technique. The cure of a small group of patients is necessary to perform two different surgeries separated by a few weeks, regardless of the type of surgery chosen the first time.

\section{Conclusion and Comments}

In this century there were two other novel coronavirus outbreaks: the 2002-2003 outbreak of severe acute respiratory syndrome (SARS) caused by SARS-CoV and the Middle East respiratory syndrome (MERS) caused by MERS-CoV, both with a high lethality rate. ${ }^{74}$ This anticipates that other outbreaks will happen shortly, mainly due to the considerable increase of the world population and the dynamic transit that exists between the various nations and continents. We must, therefore, be prepared with practical guidelines supported by the international academic community, since these outbreaks are everyone's problem and can eventually become pandemics as in the case of the Covid-19.

In conclusion, in this article, the authors suggest a set of measures that could decrease the possibility of the surgeon becoming infected when performing a surgical procedure in a patient with CSDH and Covid-19. Much of what has been shown and discussed here regarding surgery of CSDH can be used when operating patients with other neurosurgical conditions, just adapting the suggestions listed above.

With these suggestions commented on here in this article, we hope to stimulate further discussion on the topic in order to optimize the strategies to protect our neurosurgeons without significantly affecting the prognosis of our operated patients who happen to be infected with SARS-CoV-2.
Funding: The authors received no specific funding for this work. Conflict of Interest: On behalf of all authors, the corresponding author states that there is no conflict of interest.

Ethical approval: Not applicable.

Informed consent: Not applicable.

Marcelo Moraes Valença

https://orcid.org/0000-0003-0678-3782

Martina Falcão Valença

https://orcid.org/0000-0002-4085-3118

Carolina Martins

https://orcid.org/0000-0002-0197-3520

Luciana Patrízia Alves Andrade-Valença

https://orcid.org/0000-0002-3487-0325

Marcelo Andrade Valença

https://orcid.org/0000-0002-0824-0928

Ana Cristina Veiga Silva

https://orcid.org/0000-0002-1 149-4427

Hildo Rocha Cirne de Azevedo Filho

https://orcid.org/0000-0002-1555-3578

\section{References}

1. Weiss $P$, Murdoch DR. Clinical course and mortality risk of severe COVID-19. Lancet. 2020.

2. Zhou $F, Y u T, D u R$, et al. Clinical course and risk factors for mortality of adult inpatients with COVID-19 in Wuhan, China: a retrospective cohort study. Lancet. 2020.

3. SBNS. TRANSMISSION OF COVID-19 DURING NEU ROSURGICAL PROCEDURES. In: Society of British Neurological Surgeons; 2020.

4. Alhazzani W, Moller MH, Arabi YM, et al. Surviving Sepsis Campaign: Guidelines on the Management of Critically III Adults with Coronavirus Disease 2019 (COVID-19). Crit Care Med. 2020.

5. Wu Z, McGoogan JM. Characteristics of and Important Lessons From the Coronavirus Disease 2019 (COVID-19) Outbreak in China: Summary of a Report of 72314 Cases From the Chinese Center for Disease Control and Prevention. JAMA. 2020.

6. Ti LK, Ang LS, Foong TW, Ng BSW. What we do when a COVID-19 patient needs an operation: operating room preparation and guidance. Can J Anaesth. 2020.

7. Tan YT, Wang JW, Zhao K, et al. Preliminary Recommendations for Surgical Practice of Neurosurgery Department in the Central Epidemic Area of 2019 Coronavirus Infection. Curr Med Sci. 2020.

8. Bourouiba L. Turbulent Gas Clouds and Respiratory Pathogen Emissions: Potential Implications for Reducing Transmission of COVID-19. JAMA. 2020.

9. Holshue ML, DeBolt C, Lindquist S, et al. First Case of 2019 Novel Coronavirus in the United States. N Engl J Med. 2020;382:929-936.

10. Chen $L$, Li $Q$, Zheng $D$, et al. Clinical Characteristics of Pregnant Women with Covid-19 in Wuhan, China. N Engl J Med. 2020.

11. Nath A. Neurologic complications of coronavirus infections. Neurology. 2020.

12. Sahyouni R, Goshtasbi K, Mahmoodi A, Tran DK, Chen JW. Chronic Subdural Hematoma: A Historical and Clinical Perspective. World Neurosurg. 2017; 108:948953. 
13. Sahyouni R, Mahboubi H, Tran P, Roufail JS, Chen JW. Membranectomy in Chronic Subdural Hematoma: Meta-Analysis. World Neurosurg. 2017; 104:418-429.

14. Santarius T, Kirkpatrick PJ, Kolias AG, Hutchinson PJ. Working toward rational and evidence-based treatment of chronic subdural hematoma. Clin Neurosurg. 2010;57:112-122.

15. Yang W, Huang J. Chronic Subdural Hematoma: Epidemiology and Natural History. Neurosurg Clin N Am. 2017;28:205-210.

16. Bacigaluppi S, Guastalli F, Bragazzi NL, Balestrino A, Bruzzi P, Zona G. Prognostic factors in chronic subdural hematoma: results from a monocentric consecutive surgical series of 605 patients. J Neurosurg Sci. 2017.

17. Wang S, Ma Y, Zhao X, et al. Risk factors of hospital mortality in chronic subdural hematoma: A retrospective analysis of 1117 patients, a single institute experience. J Clin Neurosci. 2019;67:46-51.

18. de Araujo Silva DO, Matis GK, Costa LF, et al. Chronic subdural hematomas and the elderly: Surgical results from a series of 125 cases: Old "horses" are not to be shot! Surg Neurol Int. 2012;3:150.

19. Pang $\mathrm{CH}$, Lee $\mathrm{SE}, \mathrm{Kim} \mathrm{CH}$, et al. Acute intracranial bleeding and recurrence after bur hole craniostomy for chronic subdural hematoma. J Neurosurg. 2015;123:65-74.

20. Cenic A, Bhandari M, Reddy K. Management of chronic subdural hematoma: a national survey and literature review. Can J Neurol Sci. 2005;32:501-506.

21. Tommiska P, Lonnrot K, Raj R, Luostarinen T, Kivisaari R. Transition of a Clinical Practice to Use of Subdural Drains after Burr Hole Evacuation of Chronic Subdural Hematoma: The Helsinki Experience. World Neurosurg. 2019; 129:e614-e626.

22. Hani L, Vulcu S, Branca M, et al. Subdural versus subgaleal drainage for chronic subdural hematomas: a post hoc analysis of the TOSCAN trial. J Neurosurg. 2019:1-9.

23. Yu GJ, Han CZ, Zhang M, Zhuang HT, Jiang YG. Prolonged drainage reduces the recurrence of chronic subdural hematoma. Br J Neurosurg. 2009;23:606-611.

24. Almenawer SA, Farrokhyar F, Hong C, et al. Chronic subdural hematoma management: a systematic review and meta-analysis of 34,829 patients. Ann Surg. 2014;259:449-457.

25. Hulsbergen AFC, Yan SC, Stopa BM, et al. International practice variation in postoperative imaging of chronic subdural hematoma patients. J Neurosurg. 2018;131:1912-1919.

26. Chang SH, Yang SH, Son BC, Lee SW. Cerebellar hemorrhage after burr hole drainage of supratentorial chronic subdural hematoma. J Korean Neurosurg Soc. 2009; 46:592-595.

27. Chee CP. Exteriorization of the subdural pocket for chronic recurrent subdural hematoma. Neurosurgery. 1988;22:780-782.

28. Berghauser Pont LM, Dippel DW, Verweij BH, Dirven CM, Dammers R. Ambivalence among neurologists and neurosurgeons on the treatment of chronic subdural hematoma: a national survey. Acta Neurol Belg. 2013;1 13:55-59.

29. Court J, Touchette CJ, lorio-Morin C, Westwick HJ, Belzile F, Effendi K. Embolization of the Middle meningeal artery in chronic subdural hematoma - A systematic review. Clin Neurol Neurosurg. 2019;186:105464.

30. Dran $G$, Berthier F, Fontaine D, Rasenrarijao D, Paquis P. [Effectiveness of adjuvant corticosteroid therapy for chronic subdural hematoma: a retrospective study of 198 cases]. Neurochirurgie. 2007;53:477-482.

31. Katsigiannis S, Hamisch C, Krischek B, et al. Independent predictors for functional outcome after drainage of chronic subdural hematoma identified using a logistic regression model. J Neurosurg Sci. 2017.

32. Lu W, Wang H, Wu T, Sheng X, Ding Z, Xu G. Burr-Hole Craniostomy with T-Tube Drainage as Surgical Treatment for Chronic Subdural Hematoma. World Neurosurg. 2018; 115:e756-e760.

33. Majovsky M, Netuka D, Benes $\bigvee$, Kucera P. Burr-Hole Evacuation of Chronic Subdural Hematoma: Biophysically and Evidence-Based Technique Improvement. J Neurosci Rural Pract. 2019;10:113-118.

34. CDC. Centers for Diseases Control and Prevention Personal Protective Equipment: Questions and Answers. In; 2020.

35. Peng PWH, Ho PL, Hota SS. Outbreak of a new coronavirus: what anaesthetists should know. Br J Anaesth. 2020.

36. Wax RS, Christian MD. Practical recommendations for critical care and anesthesiology teams caring for novel coronavirus (2019-nCoV) patients. Can J Anaesth. 2020.

37. Soleman J, Nocera F, Mariani L. The conservative and pharmacological management of chronic subdural haematoma. Swiss Med Wkly. 2017; 147:w 14398.

38. Qiu S, Zhuo W, Sun C, Su Z, Yan A, Shen L. Effects of atorvastatin on chronic subdural hematoma: A systematic review. Medicine (Baltimore). 2017;96:e7290.

39. Qian Z, Yang D, Sun F, Sun Z. Risk factors for recurrence of chronic subdural hematoma after burr hole surgery: potential protective role of dexamethasone. Br J Neurosurg. 2017;31:84-88.

40. Mahmood SD, Waqas M, Baig MZ, Darbar A. Mini-Craniotomy Under Local Anesthesia for Chronic Subdural Hematoma: An Effective Choice for Elderly Patients and for Patients in a Resource-Strained Environment. World Neurosurg. 2017; 106:676-679.

41. Surve RM, Bansal S, Reddy M, Philip M. Use of Dexmedetomidine Along With Local Infiltration Versus General Anesthesia for Burr Hole and Evacuation of Chronic Subdural Hematoma (CSDH). J Neurosurg Anesthesiol. 2017;29:274-280.

42. Wang W, Feng L, Bai F, Zhang Z, Zhao Y, Ren C. The Safety and Efficacy of Dexmedetomidine vs. Sufentanil in Monitored Anesthesia Care during Burr-Hole Surgery for Chronic Subdural Hematoma: A Retrospective Clinical Trial. Front Pharmacol. 2016;7:410.

43. Zolfaghari S, Stahl N, Nittby Redebrandt H. Does time from diagnostic $C T$ until surgical evacuation affect outcome in patients with chronic subdural hematoma? Acta Neurochir (Wien). 2018; 160:1703-1709.

44. Shi S, Qin M, Shen B, et al. Association of Cardiac Injury With Mortality in Hospitalized Patients With COVID-19 in Wuhan, China. JAMA Cardiol. 2020.

45. Goldstein MR, Poland GA, Graeber CW. Are certain drugs associated with enhanced mortality in COVID-19? QJM. 2020.

46. Ji Y, Ma Z, Peppelenbosch MP, Pan Q. Potential association between COVID-19 mortality and health-care resource availability. Lancet Glob Health. 2020;8:e480.

47. Abe Y, Maruyama K, Yokoya S, et al. Outcomes of chronic subdural hematoma with preexisting comorbidities causing disturbed consciousness. J Neurosurg. 2017; 126:1042-1046. 
48. Ruan Q, Yang K, Wang W, Jiang L, Song J. Clinical predictors of mortality due to COVID-19 based on an analysis of data of 150 patients from Wuhan, China. Intensive Care Med. 2020.

49. Lei $\mathrm{S}$, Jiang $\mathrm{F}, \mathrm{Su} \mathrm{W}$, et al. Clinical characteristics and outcomes of patients undergoing surgeries during the incubation period of COVID-19 infection. EClinicalMedicine. 2020;9:1-8.

50. Schwarz F, Loos F, Dunisch P, et al. Risk factors for reoperation after initial burr hole trephination in chronic subdural hematomas. Clin Neurol Neurosurg. 2015; 138:6671.

51. Cohen-Gadol AA. Remote contralateral intraparenchymal hemorrhage after overdrainage of a chronic subdural hematoma. Int J Surg Case Rep. 2013;4:834-836.

52. Pavlov O. Rapid evacuation of chronic subdural hematoma - A possible traumatic brain injury (TBI). Med Hypotheses. 2019;137:109539.

53. Aissaoui Y, Chkoura K, Belhachmi A, Boughalem M. [Tension pneumocephalus after surgical treatment of a bilateral chronic subdural hematoma: a banal and serious complication]. Ann Fr Anesth Reanim. 2013;32:796798.

54. Akpinar A, Ucler N, Erdogan U, Yucetas CS. Epidural Hematoma Complication after Rapid Chronic Subdural Hematoma Evacuation: A Case Report. Am J Case Rep. 2015; 16:430-433.

55. Amano T, Takahara K, Maehara N, et al. Optimal perioperative management of antithrombotic agents in patients with chronic subdural hematoma. Clin Neurol Neurosurg. 2016;151:43-50.

56. Carangelo B, Lavalle L, Muscas G, et al. Internal haemorrhagic pachymeningiosis: specific disease or complication of chronic subdural hematoma? Report of five cases surgically treated and literature review. G Chir. 2014;35:190-194.

57. d'Avella D, De Blasi F, Rotilio A, Pensabene V, Pandolfo N. Intracerebral hematoma following evacuation of chronic subdural hematomas. Report of two cases. J Neurosurg. 1986;65:710-712.

58. Adachi A, Higuchi Y, Fujikawa A, et al. Risk factors in chronic subdural hematoma: comparison of irrigation with artificial cerebrospinal fluid and normal saline in a cohort analysis. PLoS One. 2014;9:e103703.

59. Bartek J, Jr., Sjavik K, Dhawan S, et al. Clinical Course in Chronic Subdural Hematoma Patients Aged 18-49 Compared to Patients 50 Years and Above: A Multicenter Study and Meta-Analysis. Front Neurol. 2019;10:311.

60. Stanišić $M$, Hald J, Rasmussen IA, et al. Volume and densities of chronic subdural haematoma obtained from CT imaging as predictors of postoperative recurrence: a prospective study of 107 operated patients. Acta Neurochir. 2013;155:323-333.

61. Chen L, Dong L, She L, et al. Treatment of chronic subdural hematoma by novel YL-1 hollow needle aspiration drainage system (697 cases report). Neurol Sci. 2017;38:109-113.
62. Fornebo I, Sjavik K, Alibeck M, et al. Role of antithrom botic therapy in the risk of hematoma recurrence and thromboembolism after chronic subdural hematoma eva cuation: a population-based consecutive cohort study. Acta Neurochir (Wien). 2017; 159:2045-2052.

63. Brennan PM, Kolias AG, Joannides AJ, et al. The management and outcome for patients with chronic subdural hematoma: a prospective, multicenter, observational cohort study in the United Kingdom. J Neurosurg. 2017:1-8.

64. Kamenova M, Lutz K, Schaedelin S, Fandino J, Mariani L, Soleman J. Does Early Resumption of Low-Dose Aspirin After Evacuation of Chronic Subdural Hematoma With Burr-Hole Drainage Lead to Higher Recurrence Rates? Neurosurgery. 2016;79:715-721.

65. Kamenova M, Mueller C, Coslovsky M, Guzman R, Mariani L, Soleman J. Low-dose aspirin and burr-hole drainage of chronic subdural hematoma: study protocol for a randomized controlled study. Trials. 2019;20:70.

66. Sim YW, Min KS, Lee MS, Kim YG, Kim DH. Recent changes in risk factors of chronic subdural hematoma. J Korean Neurosurg Soc. 2012;52:234-239.

67. Soleman J, Kamenova M, Guzman R, Mariani L. The Management of Patients with Chronic Subdural Hematoma Treated with Low-Dose Acetylsalicylic Acid: An International Survey of Practice. World Neurosurg. 2017; 107:778-788.

68. Xie $Y$, Lu Q, Lenahan C, Yang S, Zhou D, Qi X. A Comparison of Subperiosteal or Subgaleal Drainage with Subdural Drainage on the Outcomes of Chronic Subdural Hematoma: A Meta-Analysis. World Neurosurg. 2020; 135:e723-e730.

69. Gastone P, Fabrizia C, Homere M, Cacciola F, Alberto $M$, Nicola DL. Chronic subdural hematoma: results of a homogeneous series of 159 patients operated on by residents. Neurol India. 2004;52:475-477.

70. Mekaj AY, Morina AA, Mekaj YH, et al. Surgical treatment of 137 cases with chronic subdural hematoma at the university clinical center of Kosovo during the period 2008-2012. J Neurosci Rural Pract. 2015;6:186-190.

71. Wan Y, Xie D, Xue Z, et al. Single Versus Double Burr Hole Craniostomy in Surgical Treatment of Chronic Subdural Hematoma: A Meta-Analysis. World Neurosurg. 2019;131:e149-e154.

72. Stavrinou $\mathrm{P}$, Katsigiannis $\mathrm{S}$, Lee $\mathrm{JH}$, et al. Risk Factors for Chronic Subdural Hematoma Recurrence Identified Using Quantitative Computed Tomography Analysis of Hematoma Volume and Density. World Neurosurg. 2017;99:465-470.

73. Jack A, O'Kelly C, McDougall C, Findlay JM. Predicting recurrence after chronic subdural haematoma drainage. Can J Neurol Sci. 2015;42:34-39.

74. Park SE. Epidemiology, virology, and clinical features of severe acute respiratory syndrome -coronavirus-2 (SARS-CoV-2; Coronavirus Disease-19). Clin Exp Pediatr. 2020. 